\title{
Neuroprotective effect of dimethyl fumarate on cognitive impairment induced by ischemic stroke
}

\author{
Xiaowen Hou ${ }^{1,2}$, Haibin $\mathrm{Xu}^{2}$, Wanli Chen ${ }^{2}$, Nannan Zhang ${ }^{2}$, Ziai Zhao ${ }^{2}$, Xin Fang ${ }^{1}$, Xing Zhang ${ }^{1}$, \\ Huisheng Chen ${ }^{2}$, Yuanyuan $\mathrm{Xu}^{1}$ \\ ${ }^{1}$ School of Public Health, China Medical University, Shenyang 110122, China; ${ }^{2}$ Department of Neurology, General Hospital of Northern Theater \\ Command, Shenyang 110016, China \\ Contributions: (I) Conception and design: X Hou, Y Xu; (II) Administrative support: None; (III) Provision of study materials or patients: H Chen, \\ Y Xu; (IV) Collection and assembly of data: H Xu, W Chen, N Zhang; (V) Data analysis and interpretation: Z Zhao, X Fang, X Zhang; (VI) \\ Manuscript writing: All authors; (VII) Final approval of manuscript: All authors. \\ Correspondence to: Yuanyuan Xu. School of Public Health, China Medical University, No. 77 Puhe Road, Shenyang 110122 , China. \\ Email: yyxu@cmu.edu.cn; Huisheng Chen. Department of Neurology, General Hospital of Northern Theater Command, 83 Wen Hua Road, \\ Shenyang 110016, China. Email: chszh@aliyun.com.
}

Background: Oxidative damage may contribute to post-stroke cognitive impairment (PSCI), but the underlying mechanisms are not fully elucidated. Dimethyl fumarate (DMF) has been used as an antioxidant in multiple sclerosis and psoriasis patients. We hypothesized that redox state was associated with PSCI, and DMF might exert neuroprotective effect against PSCI via anti-oxidative actions.

Methods: To confirm this hypothesis, we first conducted a clinical study (NCT03519828) that enrolled patients diagnosed with acute ischemic stroke within 48 hours. Data were analyzed based on demographic characteristics, disease history, clinical data and redox state. Logistic regression was used to identify the factors associated with PSCI. Next, a middle cerebral artery occlusion (MCAO) rat model was used to explore the antioxidant capacity and neuroprotective effect of DMF. Furthermore, behavioural experiments, histology and immunostaining, and transmission electron microscopy were also performed.

Results: Higher baseline NIHSS score, lower GSH/GSSG and T-AOC levels were found in the PSCI patients. Better performance in Morris water maze and shuttle box testing, more regular arranged neurons and Nissl bodies, less TUNEL-positive cells and autophagosomes, lower expression of 4-HNE, and higher expression of GCLM and NQO1 were found in the (DMF + MCAO) rats compared with the MCAO rats.

Conclusions: These findings suggest that DMF may alleviate PSCI via neuroprotective actions, providing a new therapeutic strategy for PSCI.

Keywords: Post-stroke cognitive impairment (PSCI); dimethyl fumarate (DMF), neuroprotection; oxidative damage

Submitted Nov 12, 2019. Accepted for publication Jan 14, 2020.

doi: 10.21037/atm.2020.02.10

View this article at: http://dx.doi.org/10.21037/atm.2020.02.10 


\section{Introduction}

Stroke is the second leading cause of death in the world, resulting in more disabilities than any other diseases $(1,2)$. Besides physical impairment, cognitive impairment can be observed in many stroke patients. Post-stroke cognitive impairment (PSCI) is a category of syndromes from mild cognitive impairment to dementia induced by stroke (3), and it is one of the most common factors threatening the life quality of patients $(4,5)$. Because acute ischemic stroke (AIS) is the most common type of stroke $(6,7)$, cognitive impairment induced by AIS has attracted worldwide attention.

Ischemic stroke patients are prone to cerebral hypoxia. Prolonging the time of cerebral ischemia will aggravate the degree of hypoxia, which may lead to cognitive impairment. Frontal lobe, thalamus, hippocampus and basal ganglion are closely associated with cognitive ability, so if these brain areas are damaged, the patients are at a high risk of cognitive impairment $(8,9)$. Consequently, the time and location of cerebral ischemia usually affect cognitive ability. However, although the above conditions are similar, the cognitive ability of patients may still be different.

Some researchers suggested that oxidative damage might also contribute to PSCI (10). Sood et al. (11) have reported that ischemic stroke may cause oxidative damage, apoptosis and cognitive impairment. Loh et al. (12) have demonstrated that the increased reactive oxygen species (ROS) induced by stroke can cause neuronal injury. ROS react with cellular macromolecular through oxidation, leading to cell necrosis and apoptosis. LeDoux et al. (13) have showed that a large amount of ROS may result in cell death and neurological dysfunction.

Dimethyl fumarate (DMF), an antioxidant drug, has been approved by the U.S. Food and Drug Administration as a first-line medication for the treatment of multiple sclerosis and psoriasis $(14,15)$. Studies have reported that DMF may play a neuroprotective role through antioxidant pathways (16-18). However, DMF has never been used to treat PSCI as an antioxidant.

In this study, we first conducted a clinical study to evaluate the association between redox state and PSCI. Next, we used a middle cerebral artery occlusion (MCAO) rat model to examine whether DMF can exert neuroprotection against PSCI and explore the possible underlying mechanisms. Morris water maze and shuttle box testing were used to assess learning and memory ability. Hematoxylin and eosin (HE), Nissl dyes, terminal deoxynucleotidyl transferase-mediated dUTP nick-and labeling (TUNEL), and transmission electron microscopy were used to reflect neuronal injury. Immunofluorescent (IF) and immunohistochemical (IHC) were used to detect oxidative or anti-oxidative effect.

\section{Methods}

Standard protocol approvals, registrations, and patient consents

The protocol of our study was registered in ClinicalTrials. gov with the number NCT03519828, and it was reviewed and approved by the Ethics Committee of General Hospital of Northern Theater Command [NO. k (2017) 34]. All participants or their representatives provided written informed consent before the patients were enrolled.

\section{Participants}

Participants were gathered from General Hospital of Northern Theater Command. Inclusion criteria included: 40-80 years old, diagnosed with AIS, first stroke onset or past stroke without obvious neurological deficit $(\mathrm{mRS} \leq 2)$, time from onset to enrollment $\leq 48$ hours, no obvious aphasia or audio-visual barriers, and signed informed consent by patients or their legally authorized representatives. Exclusion criteria included: intracranial haemorrhage, transient ischemic attack, disturbance of consciousness or language expression, dementia or failure to complete assessment. The study was conducted from March 2018 to December 2018.

According to the Montreal cognitive assessment (MoCA) score, the patients was diagnosed with or without PSCI. PSCI was defined as patients with a MoCA score $<26$. If the patient's education level was middle school or below, the demarcation point was 25 (19).

\section{Data collection}

Patient information collected included demographic characteristics, disease history, clinical data and redox state. Reduced glutathione/oxidized glutathione (GSH/GSSG), total antioxidant capacity (T-AOC), catalase (CAT) and glutathione peroxidase (GSH-PX) were used as indicators of redox state in the patients. Blood samples were collected immediately after admission.

Levels of blood GSH and GSSG were measured with 
BIOXYTECG GSH/GSSG-412 kit (OxisResearch, Portland, USA). Blood with anticoagulant was immediately mixed with or without 1-methel-2-vinyl-pyridim trifluoromethane sulfonate for the measurement of GSSG and total glutathione $(\mathrm{GSH}+\mathrm{GSSG})$, respectively. $\mathrm{GSH}=$ total glutathione $-(2 \times$ GSSG).

The plasma was obtained after the centrifugation $\left(2,000 \mathrm{rpm}, 4^{\circ} \mathrm{C}, 10 \mathrm{~min}\right)$ of anticoagulant-containing blood samples. Levels of T-AOC, CAT and GSH-PX in the plasma were measured using the corresponding kits from Nanjing Jiancheng Bioengineering Institute (Nanjing, China) according to the manufacturer's instruction.

\section{Animals and bousing}

Forty-eight male Sprague-Dawley rats were obtained from Liaoning Changsheng Biotechnology Company Limited (Liaoning, China). Since most stroke patients were elderly, rats with 12-14 months of old (weighing $600 \pm 50 \mathrm{~g}$ ) were used. All the rats were housed on a twelve-hour light: twelve-hour dark cycle (6:00 a.m.-6:00 p.m.) in the Animal Experimental Centre of China Medical University, with free access to animal food and tap water ad libitum (specific pathogen-free conditions). All the animal experiments were implemented under the protocol approved by the Ethics Committee of China Medical University, and in line with the relevant guidelines and regulations.

\section{Experimental design}

A randomized 4-animal block allocation was employed to assign rats to one of the four experimental groups ( $\mathrm{n}=12$ animals/group): Sham group, DMF group, MCAO group and (DMF+ MCAO) group. Rats were numbered 1 to 48 such that for each sequential set of 4 animals, one animal in each set was allocated into each experimental group. Rats in the Sham group were pretreated with methyl cellulose (MC) and subjected to sham operation. MC was given intragastrically $12.5 \mathrm{mg} / \mathrm{kg}$ twice daily for 10 consecutive days, and 3 days before sham operation. In sham operation, right internal carotid artery (ICA), external carotid artery (ECA) and common carotid artery (CCA) were exposed, but no ligation was performed. Rats in the DMF group were pretreated intragastrically with DMF and subjected to sham operation. Similar to MC, DMF was given $12.5 \mathrm{mg} / \mathrm{kg}$ twice daily for 10 consecutive days, and 3 days before sham operation. Rats in the MCAO group were pretreated with MC and subjected to MCAO. MCAO was performed using a modified method described by Longa et al. (20). In brief, right ICA, ECA and CCA were exposed through a midline incision on the neck, and then CCA was ligated and transected. A silicone rubber cylinder was inserted into ICA until the silicone rubber cylinder was $17-19 \mathrm{~mm}$ away from CCA bifurcation. The origin of right middle cerebral artery was occluded by tightening the silicone rubber cylinder. Rats in the (DMF + MCAO) group were pretreated with DMF and subjected to MCAO. A flow diagram summarizing the process was shown in Figure $1 \mathrm{~A}$.

\section{Behavioural experiments}

Learning and memory ability and athletic ability of the rats were tested on preoperative day 1 , postoperative day 1 and postoperative day 6, respectively. Learning and memory ability was assessed by Morris water maze (MWM) and shuttle box testing. Athletic ability was tested by neurological deficit scoring. MWM testing was performed between 8 and $11 \mathrm{am}$, and shuttle box testing was performed between 2 and $5 \mathrm{pm}$.

\section{MWM testing}

MWM testing was applied to assess the ability of spatial learning and spatial memory (21). MWM task was performed in a round container $(100 \mathrm{~cm}$ in diameter and $50 \mathrm{~cm}$ in height) (Noldus Inc., Netherlands) filled with water $\left(21.5 \pm 0.5{ }^{\circ} \mathrm{C}\right)$. The pool was divided into four quadrants and monitored with a video camera on the top. A platform was placed $1 \mathrm{~cm}$ below the water surface in the third quadrant.

MWM testing began 6 days before the operation. Rats underwent 4 training trials daily for 5 continuous days. In each trial, rats were placed in one of the four quadrants to find the platform. They were allowed to search platform and climb on it within $60 \mathrm{~s}$. If the rats failed in $60 \mathrm{~s}$, they would be guided onto the platform and allowed to stay on it for $10 \mathrm{~s}$. After training trials, testing trials were performed with the platform being removed. In testing trials, rats were only placed in the quadrant opposite the platform. Testing trials were performed on preoperative day 1 , postoperative day 1 and postoperative day 6, respectively. Escape latency in the target quadrant and trajectory heat map were used as the measures for the learning and memory ability of rats. The process of MWM testing was presented in Figure $1 B$.

\section{Shuttle box testing}

Shuttle box testing was run by a two-way shuttle box 

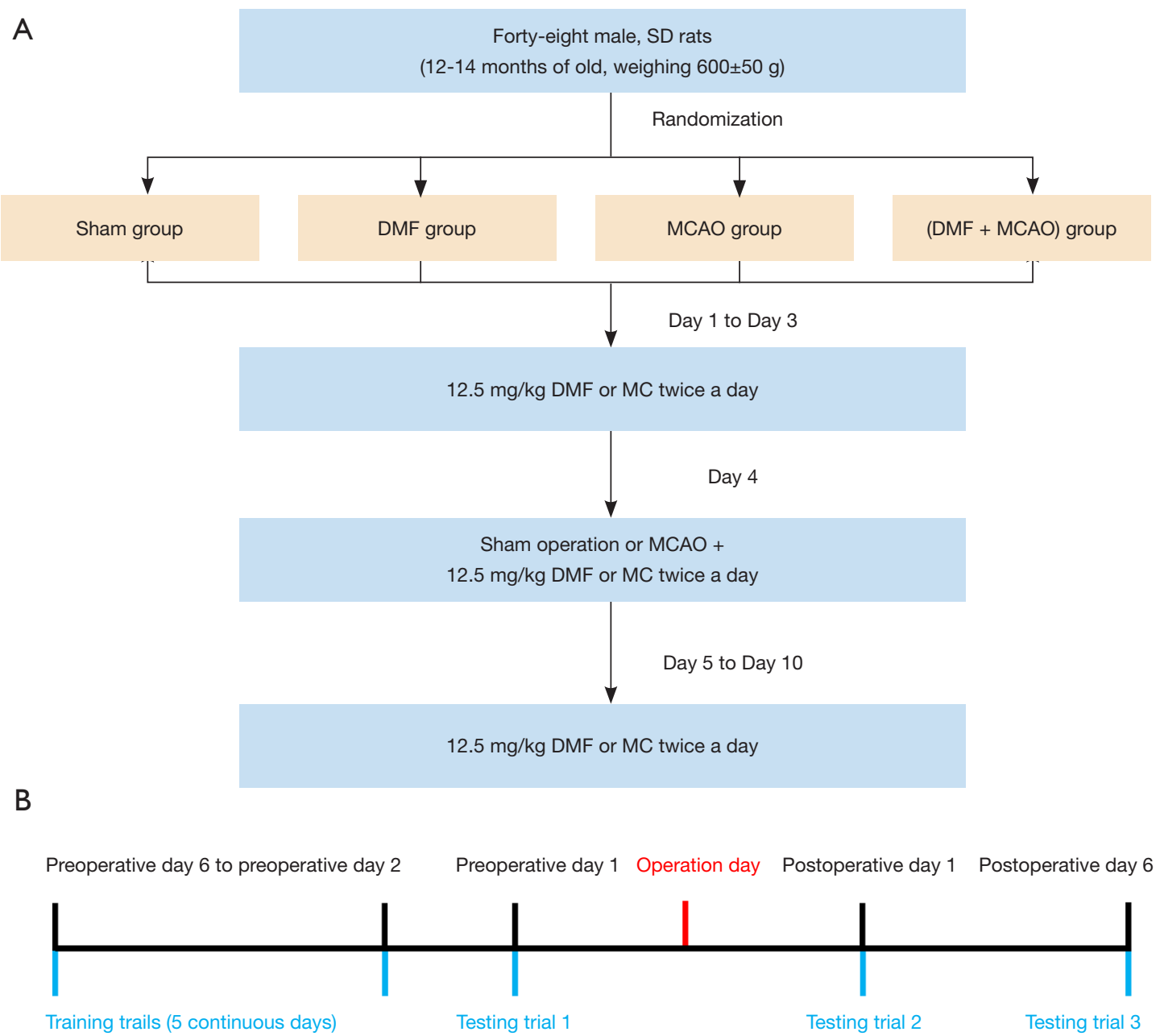

C

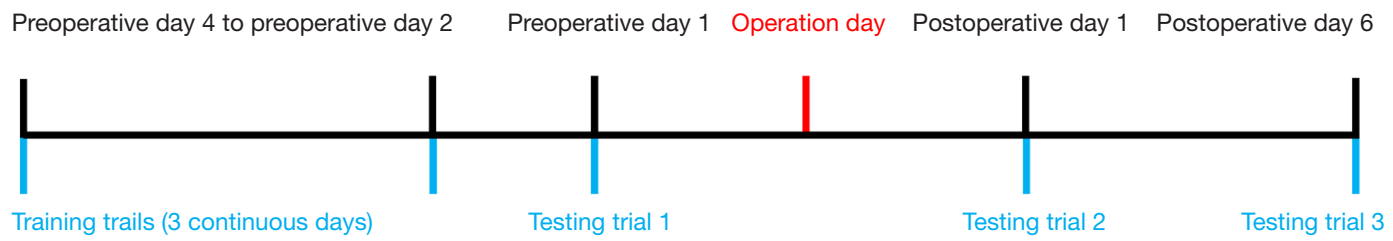

Figure 1 Flow diagram of the animal study. (A) Flow diagram summarizing the grouping and intervention design. Sham group: MC + sham operation. DMF group: DMF + sham operation. MCAO group: MC + MCAO. (DMF + MCAO) group: DMF + MCAO. Rats were pretreated with MC or DMF for 10 consecutive days, and 3 days before sham operation or MCAO; (B) flow diagram of MWM testing; (C) flow diagram of shuttle box testing.

$(30 \mathrm{~cm} \times 30 \mathrm{~cm} \times 20 \mathrm{~cm}$; Jinan Yiyan Co., Shandong, China). Training trials started 4 days before the operation and continued for 3 continuous days. Testing trials were performed on preoperative day 1 , postoperative day 1 and postoperative day 6, respectively. Each rat underwent 20 trials daily after a five-minute adaptation period. Rats were exposed to a 10 -second light followed by a 10 -second foot shock $(30 \mathrm{~V}, 1.0 \mathrm{~mA})$ in each trial. If the rat moved to the 
other chamber with the light was on, the behavior was counted as avoidance and the light was turned off with no exposure to foot shock. If it went to the other chamber with a foot shock, the behavior was counted as escape and the shock was terminated immediately. If it did not change chambers during a trial, the behavior was counted as error and the rat received a 10-second foot shock. In each trial, times of avoidance were used as the measure for the learning and memory ability. The process of shuttle box testing was presented in Figure $1 C$.

\section{Neurological deficit scoring}

Neurological deficits of rats were scored on preoperative day 1, postoperative day 1 and postoperative day 6, respectively. The neurological deficits were scored on a fivepoint scale: a score of 0 indicated no neurological deficit, a score of 1 (failure to extend left forepaw fully) indicated a mild neurological deficit, a score of 2 (circling to the left) indicated a moderate neurological deficit, a score of 3 (falling to the left) indicated a severe neurological deficit, and a score of 4 (being unable to walk spontaneously and with a depressed level of consciousness) indicated a most severe neurological deficit (20).

\section{Plasma redox state}

On the postoperative day 7 , rats were killed by decapitation after anaesthetized with $2 \%$ pentobarbital sodium for approximately 1-2 min. Levels of GSH/GSSG and T-AOC in the plasma were used as indicators of redox state in the rats. Kits from BIOXYTECG (OxisResearch, Portland, USA) and Nanjing Jiancheng Bioengineering Institute (Nanjing, China) were separately used to measure GSH/GSSG and T-AOC levels according to the manufacturer's instruction.

\section{Histology and immunostaining}

On the postoperative day 7 , rats were killed by decapitation after anaesthetized with $2 \%$ pentobarbital sodium for approximately 1-2 min. Right brain tissues were fixed in 4\% paraformaldehyde buffer overnight and then transferred to $70 \%$ ethanol. Samples were subsequently embedded in paraffin and cut into $4 \mu \mathrm{m}$ thick sections.

\section{HE staining, Nissl staining and TUNEL staining}

HE and Nissl staining were carried out according to the standard protocols. A high-power optical microscope (magnification, $\times 400$ ) was used to observe the pyramidal neurons in the hippocampal CA1 region. Three randomly selected visual fields were used to indicate the histological changes (22).

TUNEL staining was performed using an In Situ Cell Death Detection Kit, TUNEL red (Vazyme Biotech Co., Jiangsu, China), and cell nuclei were visualized with 4', 6-diamidino-2-phenylindole (DAPI) staining (Vector, Burlingame, CA, USA). TUNEL-positive cells were detected on 10 randomly selected fields from each tissue. The average number of TUNEL-positive cells in the hippocampal CA1 region per high power field was expressed as apoptosis degree (23).

\section{IF staining and IHC staining}

4-hydroxynonenal (4-HNE) was frequently measured as an indicator of oxidative damage. To examine the expression of 4-HNE in hippocampal tissue, IF staining with 4-HNE antibody (1:100; ab46545, Abcam, US) was conducted. Pretreated hippocampal sections were incubated with 4-HNE antibody at $4{ }^{\circ} \mathrm{C}$ overnight. After washing with phosphate-buffered saline, the slides were mounted with VECTASHIELD Mounting Medium. The stained sections were observed under fluorescence microscope (Nikon Corporation, Tokyo, Japan) and representative images were captured.

For IHC, rabbit polyclonal antibody against glutamate cysteine ligase modifier subunit (GCLM) (14241-1-AP, Proteintech Group, Hubei, China) and $\mathrm{NAD}(\mathrm{P}) \mathrm{H}$ : quinone oxidoreductase 1 (NQO1) (11451-1-AP, Proteintech Group, Hubei, China) were both used at dilutions of 1:200. To quantify GCLM and NQO1 expression in the tissue, ten random fields per sample were digitally imaged. All the sections were semi-quantitatively analyzed by ImagePro Plus 6.0. The integrated optical density (IOD) was measured in five fields in each section on the 400-fold magnified images, and the average IOD was used for statistical analysis (24).

\section{Transmission electron microscopy}

Hippocampal tissues were fixed in $2.5 \%$ glutaraldehyde solution (Sigma-Aldrich, C5882, St. Louis, MO) in $0.1 \mathrm{M}$ phosphate buffer overnight at $4{ }^{\circ} \mathrm{C}$. After dehydration through a graded series of ethanol, the tissues were infiltrated with Epon 812 (SPI, USA). Finally, the specimens were cut into 1- $\mu \mathrm{m}$ sections with ultramicrotome (LKB-V, Sweden) and stained with uranyl acetate and lead citrate. Images were captured by a transmission electron 
microscope (H-7650; Hitachi, Tokyo, Japan).

\section{Statistical analysis}

In the clinical study, for continuous variables that were normally distributed, data were presented as mean and standard deviation. When variables were not normally distributed, data were presented as median and interquartile range. Count data were described with $\mathrm{n}(\%)$. The Kolmogorov-Smirnov test was used to assess the normality of the data. To compare quantitative variables between the two groups, $t$-test or Wilcoxon rank sum test was used. Chisquare test was used to compare count data. Variables with a marginal association with PSCI $(\mathrm{P}<0.1)$ were entered in a multivariable logistic regression model. Multivariate binary logistic regression analyses were performed to identify the factors associated with PSCI. Odds ratio (OR) and 95\% confidence interval $(\mathrm{CI})$ were also calculated. A value of $\mathrm{P}<0.05$ in two tails was considered significant. Above data analyses were all performed with SPSS 20.0.

In the animal study, data were expressed as mean \pm standard deviation. Difference between the groups in the behavioural experiments was analyzed by repeated-measures analysis of variance followed by Bonferroni post hoc test. Similarly, a value of $\mathrm{P}<0.05$ in two tails was considered statistically significant. The statistical analyses were performed using GraphPad Prism 6.

\section{Results}

\section{Low antioxidant capacity might be associated with PSCI}

One hundred participants were enrolled in this study according to the inclusion and exclusion criteria. According to the MoCA score at admission, the participants were divided into two groups, including 61 in the PSCI group and 39 in the control group. Patient characteristics in the two groups were listed in Table 1 .

The results of the univariate analysis showed that the possible different variables between the two groups were age, stroke history, baseline National Institutes of Health Stroke Scale (NIHSS), Oxfordshire Community Stroke Project (OCSP) type, Trial of Org 10,172 in Acute Stroke Treatment (TOAST) classification, GSH/GSSG, T-AOC and GSH-PX $(\mathrm{P}<0.1)$. No significant difference was observed in the other factors between the groups. In addition, the levels of GSH/GSSG, T-AOC, CAT and GSH-PX in the groups were also showed in Figure 2.
The variables with marginal difference between the groups $(\mathrm{P}<0.1)$ were taken into multivariate logistic regression analysis. Among the variables, baseline NIHSS score, GSH/GSSG and T-AOC levels remained different between the groups $(\mathrm{P}<0.05)$. Furthermore, higher baseline NIHSS score, lower GSH/GSSG and T-AOC levels were found in the PSCI patients (Tables 2-4).

Multiplicative interaction was performed to test whether interaction existed between age and other variables which might be correlated with PSCI (stroke history, admission NIHSS, OCSP, TOAST classification, GSH/GSSG, T-AOC and GSH-PX). In this analysis, no multiplicative interaction was found between age and the variables $(\mathrm{P}>0.05)$ (Table 5). Therefore, the results in the logistic regression analysis were credible.

\section{DMF alleviated cognitive impairment of rats subjected to MCAO}

In the MWM testing, the escape latency in the training trials was shown in Figure $3 \mathrm{~A}$. Most rats could find platform quickly and accurately through the five-day training, and there was no significant difference between the groups. The escape latency of the rats on preoperative day 1 , postoperative day 1 and postoperative day 6 was shown in Figure $3 B$. The escape latency in the MCAO group was significantly longer than that in the (DMF + MCAO) group and Sham group on postoperative day 1 and postoperative day 6. The trajectory heat map was shown in Figure 3C. Multiple searches in the target quadrant were found in the groups on preoperative day 1 , and no significant difference between the groups was observed. On postoperative day 1 and postoperative day 6, searches in the target quadrant were rarely found in the MCAO group. Compared with the MCAO group, more searches in the target quadrant in the $(\mathrm{DMF}+\mathrm{MCAO})$ group and Sham group were observed.

In the shuttle box testing, the avoidance response in the training trials was shown in Figure $4 A$. An upward trend of the avoidance response was observed, and no significant difference between the groups was found. The avoidance response on preoperative day 1, postoperative day 1 and postoperative day 6 was shown in Figure 4B. On postoperative day 1 and postoperative day 6 , the avoidance response in the MCAO group was significantly less than that in the (DMF + MCAO) group and Sham group.

Neurological deficit scoring was performed in all the groups and shown in Figure 5. The neurological deficits of the rats were scored 0 in all the groups on preoperative 
Table 1 The characteristics of the participants

\begin{tabular}{|c|c|c|c|}
\hline Variables & Case $(n=61)$ & Control $(n=39)$ & $P$ \\
\hline \multicolumn{4}{|l|}{$\begin{array}{l}\text { Demographic } \\
\text { characteristics }\end{array}$} \\
\hline $\begin{array}{l}\text { Age (years), mean } \\
\pm S D\end{array}$ & $63.9 \pm 10.7$ & $58.7 \pm 13.6$ & $0.033^{*}$ \\
\hline Males, n (\%) & 45 (73.8) & $27(69.2)$ & 0.622 \\
\hline Educational level, n (\% & & & 0.121 \\
\hline $\begin{array}{l}\text { Primary school } \\
\text { degree or below }\end{array}$ & $25(41.0)$ & $10(25.6)$ & \\
\hline $\begin{array}{l}\text { Middle school } \\
\text { degree }\end{array}$ & $35(57.4)$ & $26(66.7)$ & \\
\hline $\begin{array}{l}\text { Bachelor degree or } \\
\text { above }\end{array}$ & $1(1.6)$ & $3(7.7)$ & \\
\hline $\begin{array}{l}\text { Current smoker, } \\
\text { n (\%) }\end{array}$ & $27(44.3)$ & $23(59.0)$ & 0.151 \\
\hline $\begin{array}{l}\text { Current drinker, } \\
\text { n (\%) }\end{array}$ & $13(21.3)$ & $9(23.1)$ & 0.835 \\
\hline \multicolumn{4}{|l|}{ Disease history, n (\%) } \\
\hline Hypertension & $42(68.9)$ & $32(82.1)$ & 0.142 \\
\hline Diabetes & $17(27.9)$ & $12(30.8)$ & 0.755 \\
\hline $\begin{array}{l}\text { Coronary heart } \\
\text { disease }\end{array}$ & 7 (11.5) & $1(2.6)$ & 0.145 \\
\hline Atrial fibrillation & $2(3.3)$ & $2(5.1)$ & 0.642 \\
\hline Stroke history & $21(34.4)$ & 7 (17.9) & $0.073^{*}$ \\
\hline Hyperlipemia & $26(42.6)$ & $22(56.4)$ & 0.178 \\
\hline \multicolumn{4}{|l|}{ Clinical data } \\
\hline $\begin{array}{l}\text { Admission NIHSS, } \\
\text { median (IQR) }\end{array}$ & $3[2-5]$ & $1[0-3]$ & $0.001^{*}$ \\
\hline $\begin{array}{l}\mathrm{SBP}(\mathrm{mmHg}) \\
\text { mean } \pm \mathrm{SD}\end{array}$ & $155.0 \pm 22.0$ & $153.0 \pm 20.1$ & 0.645 \\
\hline $\begin{array}{l}\mathrm{DBP}(\mathrm{mmHg}) \\
\text { mean } \pm \mathrm{SD}\end{array}$ & $90.5 \pm 12.0$ & $88.1 \pm 12.3$ & 0.333 \\
\hline $\begin{array}{l}\text { UA ( } \mu \mathrm{mol} / \mathrm{L}) \text {, } \\
\text { median (IQR) }\end{array}$ & $\begin{array}{c}303.0(275.5- \\
381.0)\end{array}$ & $\begin{array}{c}329.0(295.3- \\
394.5)\end{array}$ & 0.116 \\
\hline $\begin{array}{l}\text { Hcy }(\mu \mathrm{mol} / \mathrm{L}) \text {, } \\
\text { median (IQR) }\end{array}$ & $13.4(10.9-19.3)$ & $13.8(10.5-17.9)$ & 0.643 \\
\hline $\begin{array}{l}\mathrm{GLU}(\mathrm{mmol} / \mathrm{L}), \\
\text { mean } \pm \mathrm{SD}\end{array}$ & $6.2(5.5-7.9)$ & $6.7(5.5-9.4)$ & 0.393 \\
\hline
\end{tabular}

Table 1 (continued)
Table 1 (continued)

\begin{tabular}{lccc}
\hline Variables & Case $(n=61)$ & Control $(n=39)$ & $P$ \\
\hline $\begin{array}{l}\text { hsCRP (mg/L), } \\
\text { median (IQR) }\end{array}$ & $2.2(1.2-6.6)$ & $2.3(1.5-4.0)$ & 0.736 \\
$\begin{array}{l}\text { OAT (hours), } \\
\text { median (IQR) }\end{array}$ & $24.0(9.8-25.0)$ & $23.0(9.0-26.0)$ & 0.989 \\
OCSP type, n (\%) & & & $0.028^{*}$ \\
TACl & $1(1.6)$ & $1(2.6)$ & \\
PACl & $16(26.2)$ & $7(17.9)$ & \\
POCI & $18(29.5)$ & $4(10.3)$ & \\
LACl & $26(42.6)$ & $27(69.2)$ &
\end{tabular}

TOAST classification, $\mathrm{n}(\%)$

$0.003^{*}$

$\begin{array}{lcc}\text { LAA } & 16(26.2) & 2(5.1) \\ \text { CE } & 3(4.9) & 1(2.6) \\ \text { SVO } & 14(23.0) & 21(53.8) \\ \text { UE } & 28(45.9) & 15(38.5)\end{array}$

Redox state, mean \pm SD

$\begin{array}{lccc}\text { GSH/GSSG } & 14.5 \pm 6.0 & 18.5 \pm 7.6 & 0.004^{*} \\ \text { T-AOC }(\mathrm{U} / \mathrm{mL}) & 55.6 \pm 25.1 & 67.4 \pm 20.1 & 0.012^{*} \\ \text { CAT }(\mathrm{U} / \mathrm{mL}) & 19.0 \pm 7.3 & 17.9 \pm 7.7 & 0.477 \\ \text { GSH-PX }(\mathrm{U} / \mathrm{mL}) & 102.1 \pm 62.2 & 78.9 \pm 51.0 & 0.055^{\star}\end{array}$

*, $\mathrm{P}<0.1$. CAT, catalase; CE, cardioembolism; DBP, diastolic blood pressure; GLU, glucose; GSH/GSSG, reduced glutathione/ oxidized glutathione; GSH-PX, glutathione peroxidase; Hcy, homocysteine; hsCRP, high sensitivity $C$ reactive protein; IQR, interquartile range; LAA, large artery atherosclerosis; LACI, lacunar infarction; NIHSS, National Institutes of Health Stroke Scale; OCSP, Oxfordshire Community Stroke Project; OAT, onset-to-admission time; $\mathrm{PACl}$, partial anterior circulation infarction; $\mathrm{POCl}$, posterior circulation infarction; SBP, systolic blood pressure; SD, standard deviation; SVO, small vessel occlusion; TACI, total anterior circulation infarction; T-AOC, total antioxidant capacity; TOAST, Trial of Org 10,172 in Acute Stroke Treatment; UA, uric acid; UE, undetermined etiology. 

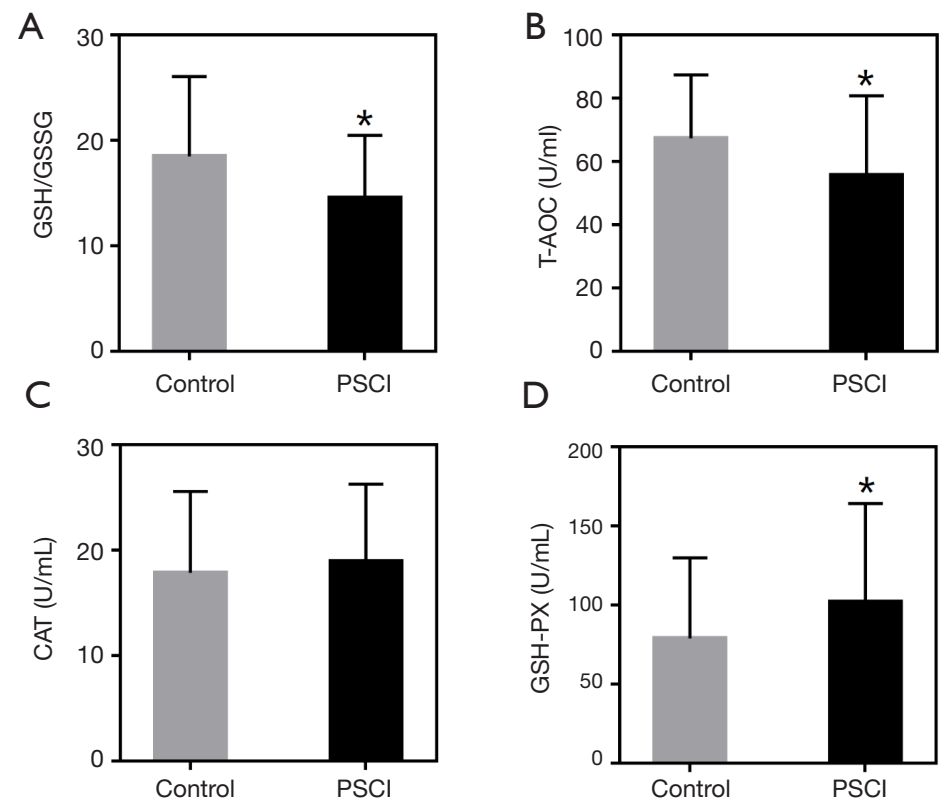

Figure 2 Redox state in the patients. Values are expressed as mean $\pm \mathrm{SD} .{ }^{*}, \mathrm{P}<0.05$, compared with the Control group. (A) Ratios of GSH to GSSG (GSH/GSSG); (B) T-AOC level; (C) CAT level; (D) GSH-PX level.

day 1 . On postoperative day 1 and postoperative day 6 , the neurological deficit scoring in the Sham group and DMF group was still 0 . There was no significant difference in the neurological deficit scoring between the MCAO group and (DMF + MCAO) group on postoperative day 1 and postoperative day 6 .

\section{DMF alleviated neuronal injury in hippocampal CA1 region of rats}

HE staining was performed to monitor neuronal morphology and pyramidal cells in hippocampal CA1 region (Figure 6A). A normal neuronal morphology with well-arranged pyramidal cells was observed in the Sham group. However, irregular neuronal arrangement with karyopyknosis and dark staining in some neuron nuclei was shown in the MCAO group. Compared with the MCAO group, more regular arranged neurons were found in the (DMF + MCAO) group.

Neuronal structure in hippocampal CA1 region was detected by Nissl staining (Figure 6B). A complete neuronal structure with clearly visible nucleoli, lightly stained cytoplasm and neat arrangement was shown in the Sham group. Nevertheless, significant cell shrinkage, irregular cell morphology, deeply stained nuclei and decreased Nissl bodies were exhibited in the MCAO group. Compared with the MCAO group, more regular cell morphology and increased Nissl bodies were shown in the (DMF + MCAO) group.

To detect the number of apoptotic cells in hippocampal CA1 region, TUNEL staining was conducted (Figure 6C). Limited TUNEL-positive cells were observed in the Sham group $(10.2 \pm 2.3)$, while dramatically more TUNELpositive cells were found in the MCAO group (37.8 \pm 5.4 ; $\mathrm{P}<0.05)$. Compared with the MCAO group, the number of TUNEL-positive cells in the (DMF + MCAO) group was less (16.2 $\pm 3.4 ; \mathrm{P}<0.05$; Figure 7).

Transmission electron microscopy imaging was also utilized to assess neuronal structure in hippocampal CA1 region (Figure 8A). None of autophagosomes was found in the Sham group. However, autophagosomes were found in the MCAO group and (DMF + MCAO) group. Compared with the MCAO group, the number of autophagosomes in the (DMF + MCAO) group was less (Figure 8B).

\section{DMF exerted anti-oxidative effect on hippocampal CA1 region of rats}

Levels of GSH/GSSG and T-AOC in the plasma were measured by the corresponding kits. There is no significant difference in GSH/GSSG and T-AOC levels between the Sham group and MCAO group. However, compared with 
Table 2 Multivariate logistic regression analysis of GSH/GSSG influence on cognitive impairment after stroke

\begin{tabular}{lccc}
\hline Variables & $\beta$ & OR $(95 \% \mathrm{Cl})$ & $\mathrm{P}$ \\
\hline Age & 0.004 & $1.004(0.959-1.051)$ & 0.863 \\
Admission NIHSS & 0.256 & $1.292(1.027-1.626)$ & $0.029^{\star *}$ \\
Previous stroke & 0.978 & $2.660(0.685-10.329)$ & 0.158 \\
TOAST classification & & & 0.230 \\
UE & - & 1 (Reference) & - \\
LAA & 1.061 & $2.891(0.521-16.024)$ & 0.224 \\
CE & 0.547 & $1.728(0.114-26.271)$ & 0.694 \\
SVO & -0.790 & $0.454(0.135-1.522)$ & 0.201 \\
OCSP type & & & 0.486 \\
LACI & - & $1($ Reference $)$ & - \\
TACI & -0.973 & $0.378(0.012-12.122)$ & 0.582 \\
PACl & -0.041 & $0.960(0.234-3.939)$ & 0.954 \\
POCI & 0.986 & $2.681(0.587-12.250)$ & 0.203 \\
GSH/GSSG & -0.089 & $0.915(0.842-0.995)$ & $0.037^{* *}$ \\
\hline
\end{tabular}

**, $\mathrm{P}<0.05$. CE, cardioembolism; $\mathrm{Cl}$, confidence interval; $\mathrm{GSH} /$ GSSG, reduced glutathione/oxidized glutathione; LAA, large artery atherosclerosis; LACI, lacunar infarction; NIHSS, National Institutes of Health Stroke Scale; OCSP, Oxfordshire Community Stroke Project; OR, odds ratio; $\mathrm{PACl}$, partial anterior circulation infarction; POCI, posterior circulation infarction; SVO, small vessel occlusion; TACl, total anterior circulation infarction; TOAST, Trial of Org 10,172 in Acute Stroke Treatment; UE, undetermined etiology.

the Sham group and MCAO group, the T-AOC level of the (DMF + MCAO) group was significantly higher (Figure 9A). Although no significant difference was found between the groups in GSH/GSSG, a reasonable trend was shown (Figure 9B).

The expression of 4-HNE was evaluated by IF staining. As shown in Figure 10A, limited expression was observed in the Sham group, while the expression was higher in the MCAO group. Besides, the expression of the protein was significantly lower in the (DMF + MCAO) group compared with the MCAO group.

The expression of GCLM and NQO1 was evaluated by IHC and shown in Figure $10 B$ and C. Compared with the Sham group, the expression was higher in the MCAO group and (DMF + MCAO) group. Additionally, the average IOD of the (DMF + MCAO) group (GCLM: 76.3 \pm 18.7 ; NQO1: $11.6 \pm 3.9)$ was higher than the MCAO group
Table 3 Multivariate logistic regression analysis of T-AOC influence on cognitive impairment after stroke

\begin{tabular}{|c|c|c|c|}
\hline Variables & $\beta$ & OR $(95 \% \mathrm{Cl})$ & $\mathrm{P}$ \\
\hline Age & 0.001 & $1.000(0.955-1.046)$ & 0.989 \\
\hline Admission NIHSS & 0.272 & $1.313(1.047-1.648)$ & $0.019^{\star \star}$ \\
\hline Previous stroke & 1.335 & 3.798 (0.913-15.799) & 0.066 \\
\hline TOAST classification & & & 0.293 \\
\hline UE & - & 1 (Reference) & - \\
\hline LAA & 0.611 & $1.842(0.311-10.897)$ & 0.501 \\
\hline CE & -0.006 & $0.994(0.077-12.772)$ & 0.996 \\
\hline SVO & -0.933 & $0.394(0.118-1.307)$ & 0.128 \\
\hline OCSP type & & & 0.374 \\
\hline $\mathrm{LACl}$ & - & 1 (Reference) & - \\
\hline $\mathrm{TACl}$ & -0.949 & $0.387(0.012-12.137)$ & 0.589 \\
\hline $\mathrm{PACl}$ & 0.087 & $1.091(0.257-4.636)$ & 0.906 \\
\hline $\mathrm{POCl}$ & 1.235 & $3.439(0.713-16.591)$ & 0.124 \\
\hline T-AOC & -0.024 & $0.976(0.954-0.999)$ & $0.038^{\star \star}$ \\
\hline
\end{tabular}

**, $\mathrm{P}<0.05$. CE, cardioembolism; $\mathrm{Cl}$, confidence interval; LAA, large artery atherosclerosis; LACI, lacunar infarction; NIHSS, National Institutes of Health Stroke Scale; OCSP, Oxfordshire Community Stroke Project; OR, odds ratio; PACI, partial anterior circulation infarction; $\mathrm{POCl}$, posterior circulation infarction; SVO, small vessel occlusion; $\mathrm{TACl}$, total anterior circulation infarction; TOAST, Trial of Org 10,172 in Acute Stroke Treatment; UE, undetermined etiology.

(GCLM: 44.5 \pm 13.2 ; NQO1: 7.5 $\pm 2.3 ; \mathrm{P}<0.05$ ), indicating the expression in the (DMF + MCAO) group was higher.

\section{Discussion}

To our knowledge, this is the first study to simultaneously conduct a clinical research and an animal study to explore the effect of antioxidation on cognitive impairment induced by ischemic stroke. First, we found that low antioxidant capacity might contribute to PSCI, which was consistent with the previous findings $(25-27)$. Next, we found that DMF might exert neuroprotective effect against PSCI via anti-oxidative actions. Although DMF has previously been reported to be effective in the treatment of ischemic stroke $(28,29)$, our study first reported the application of DMF in cognitive impairment induced by ischemic stroke.

In the clinical study, higher baseline NIHSS score, lower 
Table 4 Multivariate logistic regression analysis of GSH-PX influence on cognitive impairment after stroke

\begin{tabular}{lccc}
\hline Variables & $\beta$ & OR $(95 \% \mathrm{Cl})$ & $\mathrm{P}$ \\
\hline Age & 0.001 & $1.001(0.957-1.046)$ & 0.978 \\
Admission NIHSS & 0.268 & $1.308(1.039-1.647)$ & $0.022^{\star \star}$ \\
Previous stroke & 0.950 & $2.585(0.703-9.510)$ & 1.153 \\
TOAST classification & & & 0.161 \\
UE & - & 1 (Reference) & - \\
LAA & 1.093 & $2.983(0.536-16.581)$ & 0.212 \\
CE & 0.412 & $1.510(0.117-19.520)$ & 0.752 \\
SVO & -0.904 & $0.405(0.123-1.338)$ & 0.138 \\
OCSP type & & & 0.647 \\
LACI & - & $1($ Reference $)$ & - \\
TACl & -0.888 & $0.411(0.008-20.386)$ & 0.656 \\
PACl & -0.019 & $0.981(0.240-4.004)$ & 0.979 \\
POCI & 0.848 & $2.336(0.497-10.981)$ & 0.283 \\
GSH-PX & 0.006 & $1.006(0.997-1.014)$ & 0.204 \\
\hline
\end{tabular}

**, $\mathrm{P}<0.05$. CE, cardioembolism; $\mathrm{Cl}$, confidence interval; $\mathrm{GSH}-$ $\mathrm{PX}$, glutathione peroxidase; LAA, large artery atherosclerosis; LACI, lacunar infarction; NIHSS, National Institutes of Health Stroke Scale; OCSP, Oxfordshire Community Stroke Project; $\mathrm{OR}$, odds ratio; $\mathrm{PACl}$, partial anterior circulation infarction; $\mathrm{POCl}$, posterior circulation infarction; SVO, small vessel occlusion; $\mathrm{TACl}$, total anterior circulation infarction; TOAST, Trial of Org 10,172 in Acute Stroke Treatment; UE, undetermined etiology.

GSH/GSSG and T-AOC levels were found in the PSCI patients. NIHSS score is a reliable, valid and responsive tool for measuring stroke severity (30). Previous studies have reported that cognitive ability was closely related to the severity of stroke $(31,32)$, which was consistent with our findings. GSH/GSSG and T-AOC levels are both recognized as indicators of antioxidant capacity (33-36). In the present study, low antioxidant capacity was considered to be associated with PSCI. Due to $20 \%$ of the body's oxygen consumption and insufficient antioxidant defense, the brain is vulnerable to oxidative damage (37). Some studies have shown that oxidative damage could cause neuronal cells death, leading to PSCI $(38,39)$, which may explain our conclusion. Previous studies have reported that age may be an independent risk factor for PSCI (40-42). In our study, the univariate analysis results showed age was a possible risk factor for PSCI, but the multivariate logistic regression analysis results suggested no significant association between
Table 5 Logistic regression analysis of interaction of age and other factors

\begin{tabular}{lccc}
\hline Interactive variables & $\beta$ & $\mathrm{OR}(95 \% \mathrm{Cl})$ & $\mathrm{P}$ \\
\hline Age and stroke history & & & \\
Age & 0.022 & $1.023(0.983-1.064)$ & 0.266 \\
Stroke history & -3.058 & $0.047(0.000-91.865)$ & 0.429 \\
$\begin{array}{l}\text { Age-stroke history } \\
\text { interaction }\end{array}$ & 0.056 & $1.057(0.942-1.187)$ & 0.344
\end{tabular}

Age and admission NIHSS

$\begin{array}{lccc}\text { Age } & 0.014 & 1.014(0.965-1.067) & 0.580 \\ \text { Admission NIHSS } & -0.193 & 0.825(0.265-2.570) & 0.740 \\ \begin{array}{l}\text { Age-admission NIHSS } \\ \text { interaction }\end{array} & 0.007 & 1.007(0.989-1.026) & 0.432\end{array}$

Age and OCSP

Age $\quad-0.064 \quad 0.938(0.776-1.133) \quad 0.507$

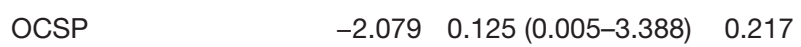

Age-OCSP interaction $\quad 0.027 \quad 1.027(0.976-1.082) \quad 0.306$

Age and TOAST

classification

$\begin{array}{lrcc}\text { Age } & 0.105 & 1.110(0.988-1.248) & 0.080 \\ \text { TOAST classification } & 0.981 & 2.667(0.443-16.050) & 0.284 \\ \begin{array}{l}\text { Age-TOAST classification } \\ \text { interaction }\end{array} & 0.018 & 0.982(0.955-1.011) & 0.222 \\ & & \end{array}$

Age and GSH/GSSG

$\begin{array}{lccc}\text { Age } & 0.001 & 1.001(0.891-1.124) & 0.990 \\ \text { GSH/GSSG } & -0.262 & 0.770(0.491-1.207) & 0.254 \\ \begin{array}{l}\text { Age-GSH/GSSG } \\ \text { interaction }\end{array} & 0.002 & 1.002(0.996-1.009) & 0.471\end{array}$

Age and T-AOC

$\begin{array}{lccc}\text { Age } & 0.097 & 1.102(0.970-1.252) & 0.137 \\ \text { T-AOC } & 0.031 & 1.032(0.921-1.156) & 0.589 \\ \text { Age-T-AOC interaction } & -0.001 & 0.999(0.997-1.001) & 0.347\end{array}$

Age and GSH-PX

\begin{tabular}{lrrr} 
Age & 0.080 & $1.083(0.989-1.186)$ & 0.087 \\
GSH-PX & 0.163 & $1.177(0.885-1.565)$ & 0.263 \\
Age-GSH-PX interaction & -0.002 & $0.998(0.993-1.002)$ & 0.300 \\
\hline
\end{tabular}

$\mathrm{Cl}$, confidence interval; GSH/GSSG, reduced glutathione/ oxidized glutathione; GSH-PX, glutathione peroxidase; NIHSS, National Institutes of Health Stroke Scale; OCSP, Oxfordshire Community Stroke Project; OR, odds ratio; T-AOC, total antioxidant capacity; TOAST, Trial of Org 10,172 in Acute Stroke Treatment. 

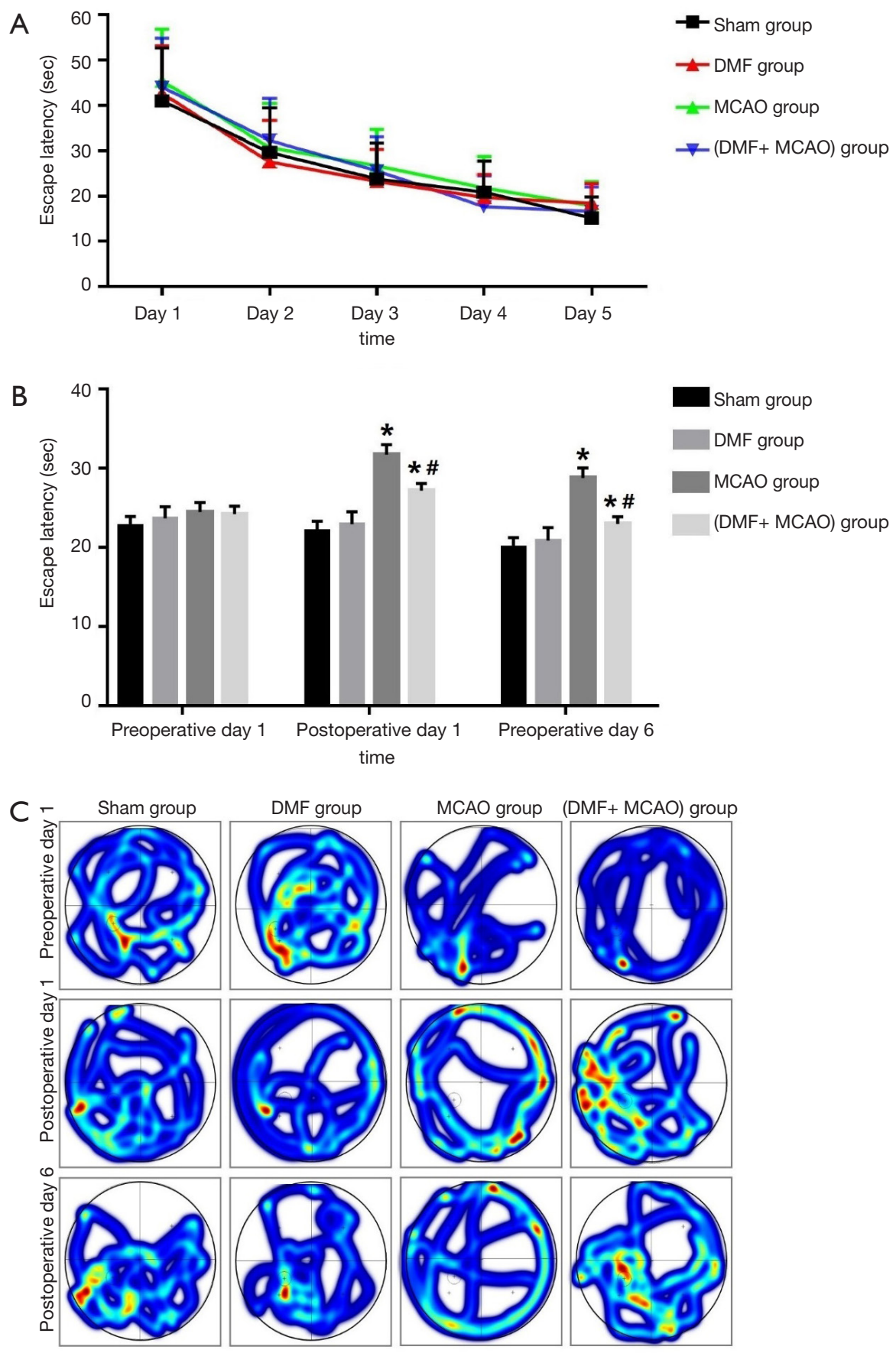

Figure 3 Escape latency and trajectory heat map of the rats in the MWM testing. Values are expressed as mean $\pm \mathrm{SD}$. * $\mathrm{P}<0.05$, compared with the Sham group; * $\mathrm{P}<0.05$, compared with the MCAO group. $\mathrm{n}=8-10$. (A) The escape latency for 5 continuous days in the training trials; (B) the escape latency on preoperative day 1, postoperative day 1 and postoperative day 6 ; (C) trajectory heat map on preoperative day 1 , postoperative day 1 and postoperative day 6 . 

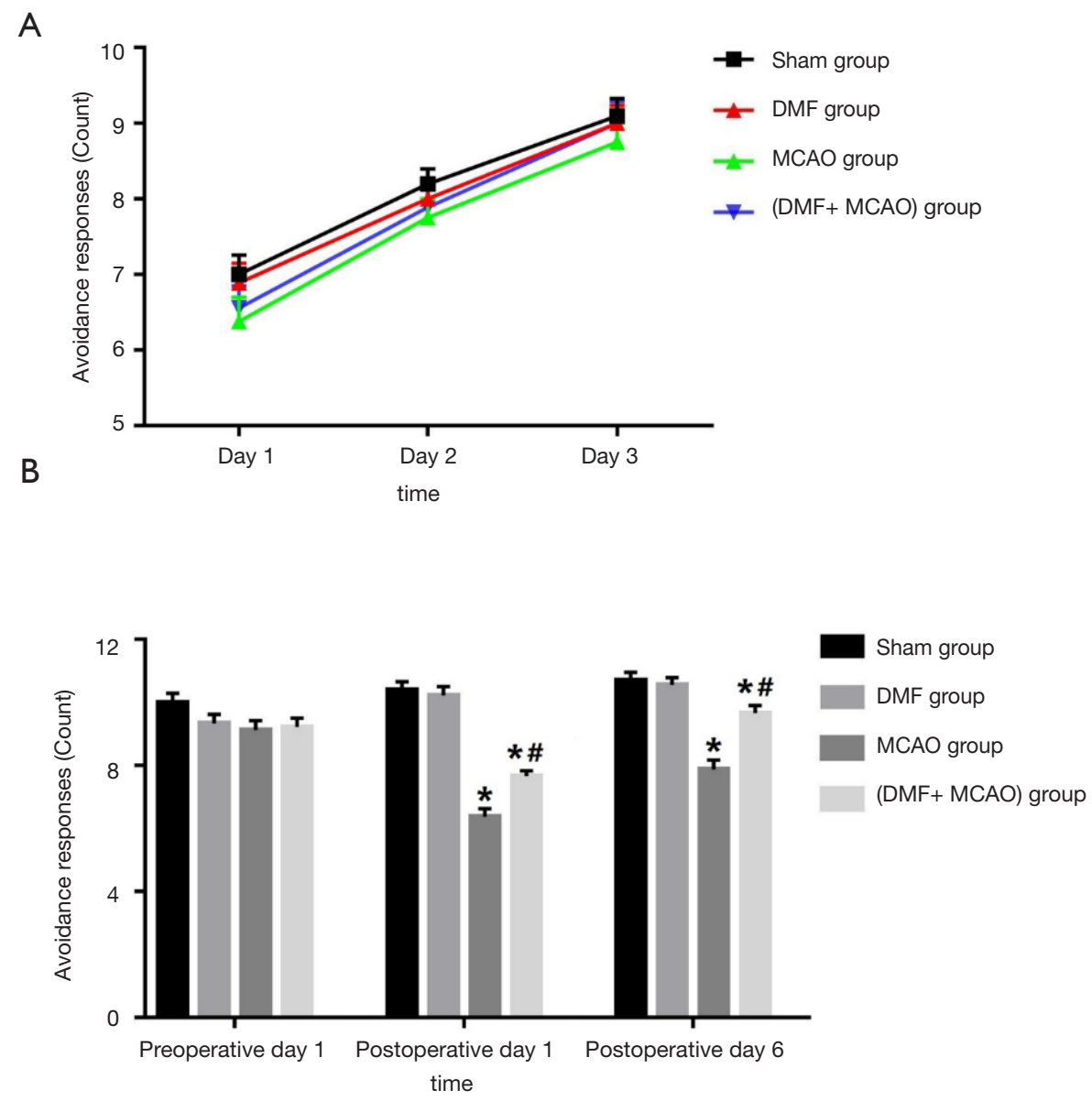

Figure 4 Avoidance responses of the rats in the shuttle box testing. Values are expressed as mean $\pm \mathrm{SD}$. *, $\mathrm{P}<0.05$, compared with Sham group; \#, $\mathrm{P}<0.05$, compared with MCAO group. $\mathrm{n}=8-10$. (A) Avoidance responses for 3 continuous days in the training trials; (B) avoidance responses on preoperative day 1 , postoperative day 1 and postoperative day 6.

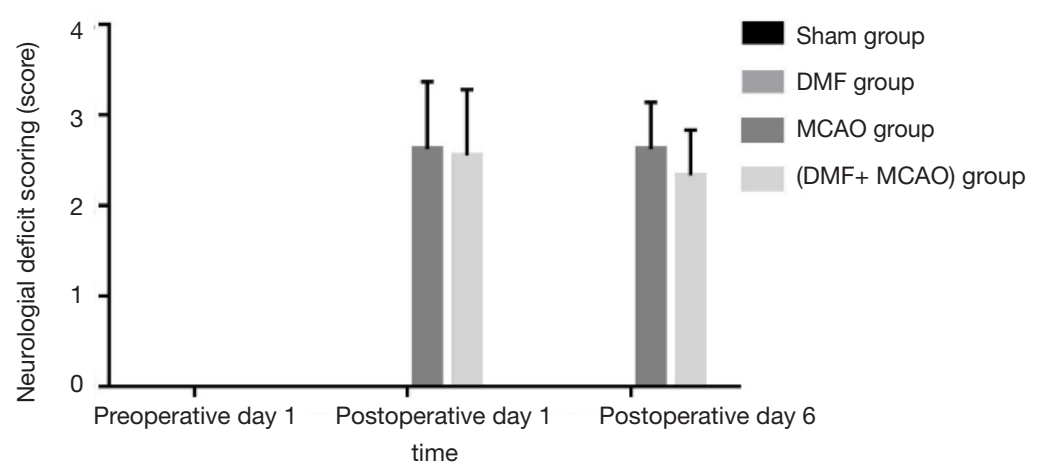

Figure 5 Neurological deficit scoring of the rats. Values are expressed as mean \pm SD. $n=8-10$. The neurological deficits were scored 0 in the four groups on preoperative day 1. On postoperative day 1 and postoperative day 6, the neurological deficits in the Sham group and DMF group were still 0, while neurological deficit scoring increased in the MCAO group and (DMF + MCAO) group. 


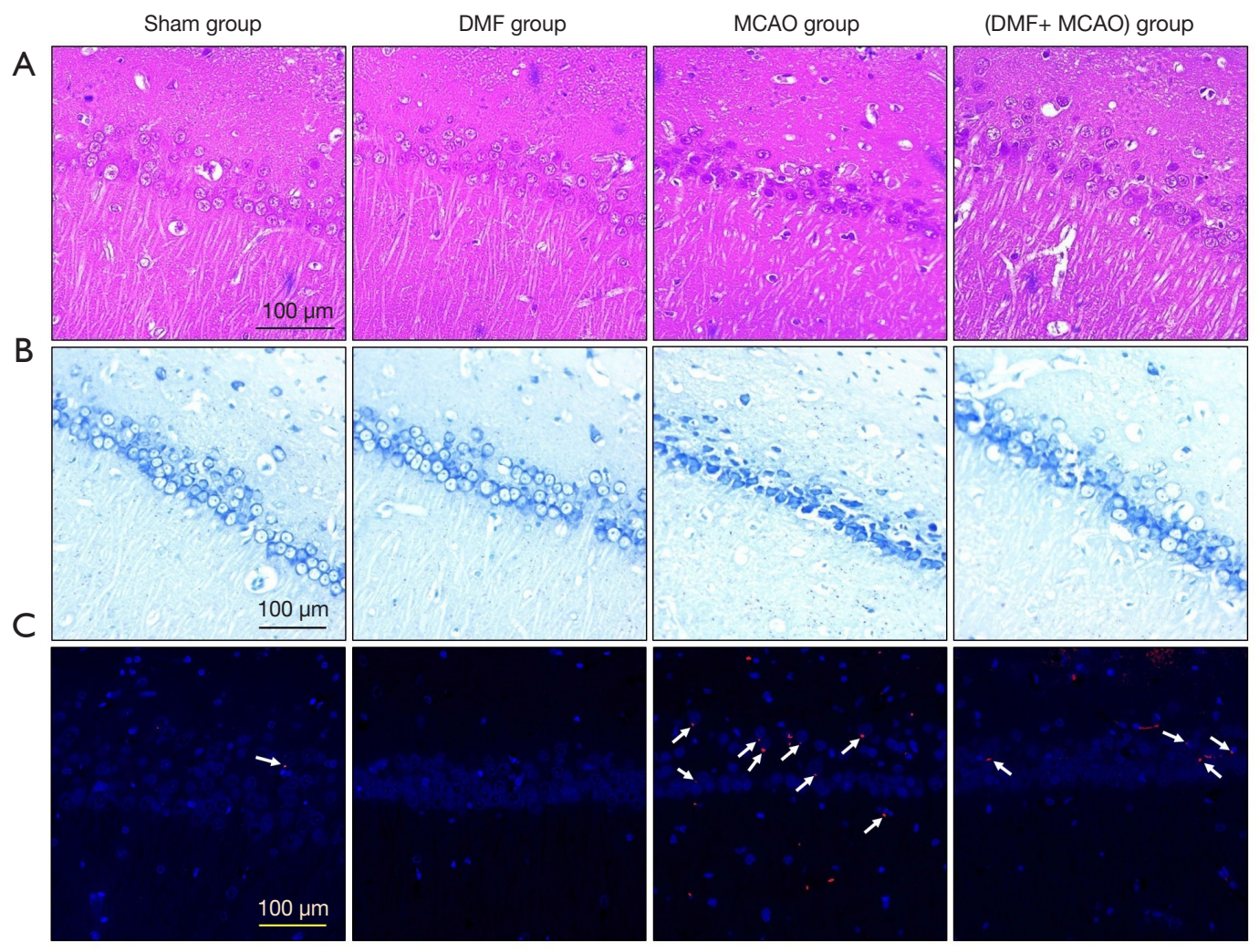

Figure $6 \mathrm{DMF}$ alleviates neuronal injury in hippocampal CA1 region. n=4-6. (A) H\&E staining and (B) Nissl staining were conducted to observe neuronal morphology (magnification $\times 400$ ); (C) TUNEL staining was conducted to observe apoptotic cells (magnification $\times 200$ ).

age and PSCI. To reach a more accurate conclusion, studies with larger sample size are needed in the future.

Corresponding to the elderly stroke patients in the clinical study, Sprague-Dawley rats (12-14 months of old) were used in the animal study. In the MWM and shuttle box testing, better performance was found in the (DMF + $\mathrm{MCAO}$ ) rats compared with the MCAO rats. Combined with the similar neurological deficit scoring in the two groups, DMF was considered to improve the learning and memory ability of the MCAO rats. Histopathological morphology of the neurons in the hippocampal CA1 region was observed by $\mathrm{HE}$ and Nissl staining. Compared with the MCAO rats, more regular arranged neurons and Nissl bodies were observed in the (DMF + MCAO) rats. Furthermore, the less number of the TUNEL-positive cells and autophagosomes in the (DMF + MCAO) rats suggested DMF might reduce cell apoptosis and autophagy. Overall, DMF might play a neuroprotective role in the cognitive impairment induced by ischemic stroke.

4-HNE is a major end-product of oxidation of polyunsaturated fatty acids, and it is usually considered as an indicator of oxidative damage. Lipid peroxides and their decomposition products can directly or indirectly affect many functions necessary to maintain homeostasis in cells and organs $(43,44)$. In the present study, the expression of 4-HNE was lower in the (DMF + MCAO) rats compared with the MCAO rats, which suggested that DMF might alleviate oxidative damage in the MCAO rats. Nuclear factor erythroid 2-related factor $2(\mathrm{Nrf2})$ is the major regulator of the cellular defense machinery against oxidative stress, and it regulates the expression of the downstream genes through the antioxidant response element (ARE) (45). Antioxidant enzymes (such as GCLM) and phase II drug metabolizing enzymes (such as NQO1) are both encoded by the downstream genes (46). The expression of GCLM and NQO1 was higher in the MCAO group and (DMF + MCAO) group compared with the Sham group. That was because the rats subjected to MCAO suffered from oxidative damage, resulting in the increase of anti-oxidative effect. Furthermore, the expression of GCLM and NQO1 was 
higher in the (DMF + MCAO) group compared with the MCAO group, suggesting DMF might exert anti-oxidative effect via the ARE of Nrf2.

Although our study shows DMF may play a neuroprotective role in the patients with PSCI, the side effects of DMF should also be taken seriously. DMF is an alkylating agent, which can modify nucleophilic groups in proteins (such as cysteine thiol) nonspecifically and covalently $(47,48)$. As a result, DMF may have serious side effects. For instance, a 30\% decline in lymphocytes has occurred after taking DMF, which may lead to infection

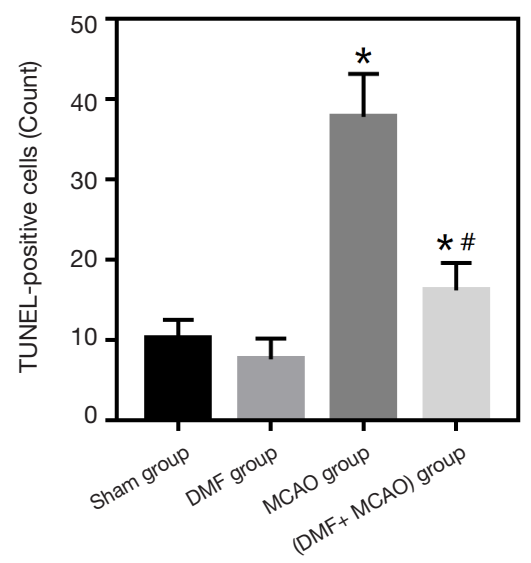

Figure 7 Quantitation of TUNEL-positive cells. Values are expressed as mean $\pm \mathrm{SD} .{ }^{*}, \mathrm{P}<0.05$, compared with the Sham group; ${ }^{\#}, \mathrm{P}<0.05$, compared with the MCAO group. $\mathrm{n}=4-6$.
$(49,50)$. Furthermore, since rebound phenomena after discontinuation of DMF for multiple sclerosis have previously been described (51), further researches should be conducted to determine the long-term anti-oxidant effect on PSCI. Metabolite monomethyl fumarate (MMF) is the major metabolite of DMF. The ester's structure of MMF enables it rapidly come across the blood-brain barrier to implement direct anti-oxidant effects on nerve cells $(52,53)$. In recent years, because the electrophilicity of MMF is weaker than that of DMF, researchers began to pay attention to MMF with the hope of developing a safer drug than DMF $(54,55)$.

Some limitations should be addressed. First, the sample size is relatively small in the clinical study. To reach a more definitive conclusion, studies with larger sample size are needed in the future. Second, because depression, anxiety, and poor sleep quality have not been measured, their relationship with PSCI could not be assessed. In order to obtain more comprehensive factors affecting PSCI, these factors need to be measured. Third, patients in the study received similar routine treatments such as antiplatelet, anticoagulant and statin according to the Chinese stroke guideline. However, we did not measure those redox levels after treatment in the present study, thus the dynamic effect of those redox levels has not determined. Also, we did not follow up the patients in the present study, so we only measured the cognitive ability at admission and used the MoCA score at admission to reflect the cognitive ability after stroke. In order to explore the long-term relationship between redox level and PSCI, follow-up should be

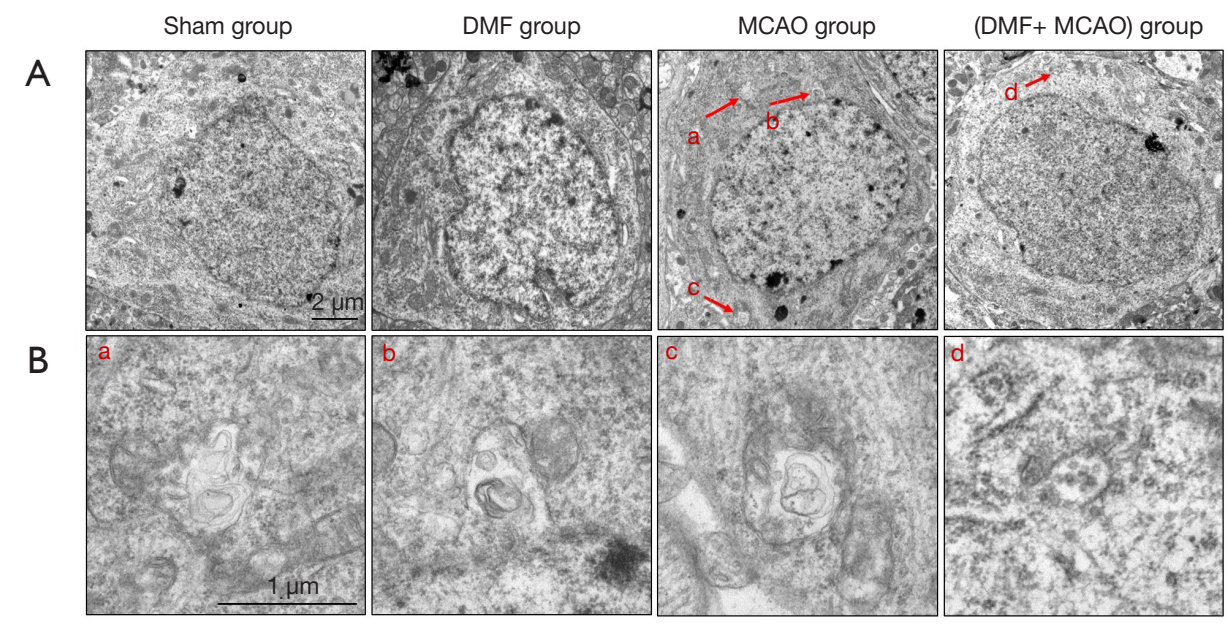

Figure 8 Autophagesomes observed by transmission electron microscopy. Small letters in Figure $8 A$ and $B$ indicate autophagosomes and the corresponding amplified autophagosomes, respectively. (A) Transmission electron microscopy was used to observe autophagesomes (magnification $\times 10,000)$; (B) the amplified autophagosomes in (A) (magnification $\times 25,000)$. 

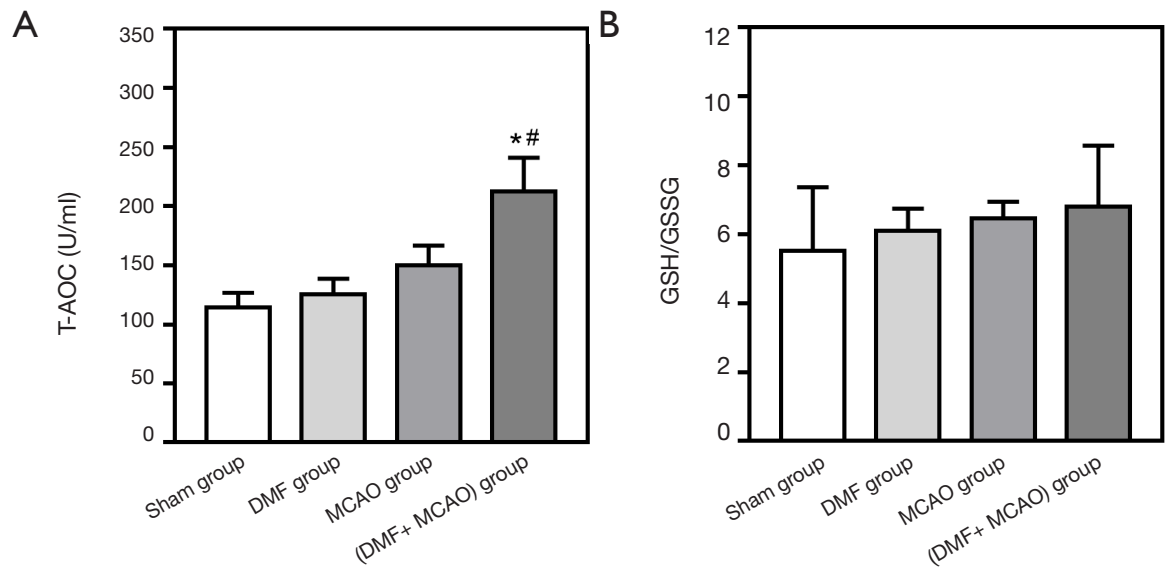

Figure 9 Redox state in the rats. Values are expressed as mean $\pm \mathrm{SD}$. *, $\mathrm{P}<0.05$, compared with the Sham group; ${ }^{*}, \mathrm{P}<0.05$, compared with the MCAO group. n=8-10. (A) T-AOC level; (B) ratios of GSH to GSSG (GSH/GSSG).

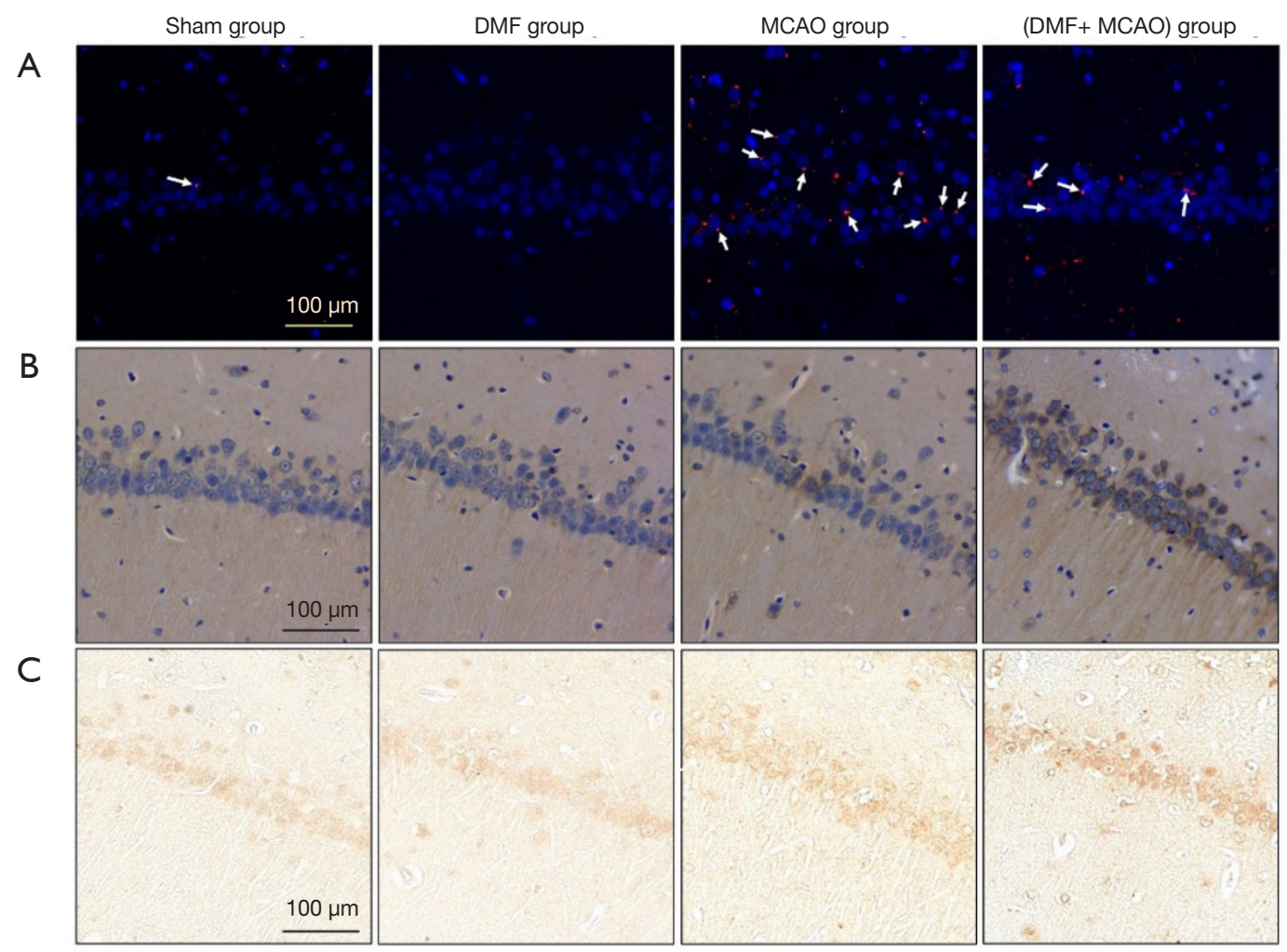

Figure 10 DMF exerts anti-oxidative effect on hippocampal CA1 region. $n=4-6$. (A) The expression of 4-HNE was evaluated by IF staining (magnification $\times 200$ ); (B) the expression of GCLM was evaluated by IHC (magnification $\times 400$ ); (C) the expression of NQO1 was evaluated by IHC (magnification $\times 400)$. 
conducted. Finally, DMF may also play a neuroprotective role through other mechanisms, such as anti-inflammatory response, so the specific mechanism needs to be further explored.

In summary, our study suggested that low antioxidant capacity might be associated with PSCI, and DMF might alleviate PSCI via neuroprotective actions, providing a new therapeutic strategy for PSCI. Further researches are needed to explore the underlying mechanisms.

\section{Acknowledgments}

We would like to thank Dr. Mingdan You and Dr. Liang Liu for their technical support.

Funding: The study was supported by the Chinese Nature Science Foundation (81573187) to Prof. Yuanyuan Xu and the grants from the Science and Technology Project Plan of Liao Ning Province (2018225023, 2019JH2/10300027) to Dr. Huisheng Chen.

\section{Footnote}

Conflicts of Interest: The authors have no conflicts of interest to declare.

Ethical Statement: The authors are accountable for all aspects of the work in ensuring that questions related to the accuracy or integrity of any part of the work are appropriately investigated and resolved. The clinical study was reviewed and approved by the Ethics Committee of General Hospital of Northern Theater Command [NO. k (2017) 34]. Written informed consent was obtained from all patients. The animal study was approved by the Ethics Committee of China Medical University.

Open Access Statement: This is an Open Access article distributed in accordance with the Creative Commons Attribution-NonCommercial-NoDerivs 4.0 International License (CC BY-NC-ND 4.0), which permits the noncommercial replication and distribution of the article with the strict proviso that no changes or edits are made and the original work is properly cited (including links to both the formal publication through the relevant DOI and the license). See: https://creativecommons.org/licenses/by-nc-nd/4.0/.

\section{References}

1. World Health Organization. The top 10 causes of death.
Available online: http://www.who.int/mediacentre/ factsheets/fs310/en/\#.WtYfmzIk_Jk.mendeley, accessed 24 May 2018.

2. Adamson J, Beswick A, Ebrahim S. Is stroke the most common cause of disability? J Stroke Cerebrovasc Dis 2004:13:171-7.

3. Leys D, Hénon H, Mackowiak-Cordoliani MA, et al. Poststroke dementia. Lancet Neurol 2005;4:752-9.

4. Swartz RH, Bayley M, Lanctôt KL, et al. Post-stroke depression, obstructive sleep apnea, and cognitive impairment: Rationale for, and barriers to, routine screening. Int J Stroke 2016;11:509-18.

5. Patel MD, Coshall C, Rudd AG, et al. Cognitive impairment after stroke: clinical determinants and its associations with long-term stroke outcomes. J Am Geriatr Soc 2002;50:700-6.

6. Wang W, Jiang B, Sun H, et al. Prevalence, incidence, and mortality of stroke in China: results from a nationwide population-based survey of 480687 adults. Circulation 2017;135:759-71.

7. Wang D, Liu J, Liu M, et al. Patterns of stroke between university hospitals and nonuniversity hospitals in Mainland China: prospective multicenter hospital-based registry study. World Neurosurg 2017;98:258-65.

8. Kandiah N, Wiryasaputra L, Narasimhalu K, et al. Frontal subcortical ischemia is crucial for post stroke cognitive impairment. J Neurol Sci 2011;309:92-5.

9. Myers CE, Shohamy D, Gluck MA, et al. Dissociating hippocampal versus basal ganglia contributions to learning and transfer. J Cogn Neurosci 2003;15:185-93.

10. Castagne V, Gautschi M, Lefevre K, et al. Relationships between neuronal death and the cellular redox status. Focus on the developing nervous system. Prog Neurobiol 1999;59:397-423.

11. Sood A, Mehrotra A, Dhawan DK, et al. Indian Ginseng (Withania somnifera) supplementation ameliorates oxidative stress and mitochondrial dysfunctions in experimental model of stroke. Metab Brain Dis 2018;33:1261-74.

12. Loh KP, Huang SH, De Silva R, et al. Oxidative stress: apoptosis in neuronal injury. Curr Alzheimer Res 2006;3:327-37.

13. LeDoux SP, Druzhyna NM, Hollensworth SB, et al. Mitochondrial DNA repair: a critical player in the response of cells of the CNS to genotoxic insults. Neuroscience 2007;145:1249-59.

14. Bomprezzi R. Dimethyl fumarate in the treatment of relapsing-remitting multiple sclerosis: an overview. Ther 
Adv Neurol Disord 2015;8:20-30.

15. Mrowietz U, Asadullah K. Dimethylfumarate for psoriasis: more than a dietary curiosity. Trends Mol Med 2005;11:43-8.

16. Ashrafian H, Czibik G, Bellahcene M, et al. Fumarate is cardioprotective via activation of the Nrf2 antioxidant pathway. Cell Metab 2012;15:361-71.

17. Sheremata $W$, Brown AD, Rammohan KW. Dimethyl fumarate for treating relapsing multiple sclerosis. Expert Opin Drug Saf 2015;14:161-70.

18. Linker RA, Lee DH, Ryan S, et al. Fumaric acid esters exert neuroprotective effects in neuroinflammation via activation of the Nrf2 antioxidant pathway. Brain 2011;134:678-92.

19. Nasreddine ZS, Phillips NA, Bédirian V, et al. The Montreal Cognitive Assessment, MoCA: a brief screening tool for mild cognitive impairment. J Am Geriatr Soc 2005;53:695-9.

20. Longa EZ, Weinstein PR, Carlson S, et al. Reversible middle cerebral artery occlusion without craniectomy in rats. Stroke 1989;20:84-91.

21. Morris RG, Garrud P, Rawlins JN, et al. Place navigation impaired in rats with hippocampal lesions. Nature 1982;297:681-3.

22. Ji S, Li S, Zhao X, et al. Protective role of phenylethanoid glycosides, Torenoside B and Savatiside A, in Alzheimer's disease. Exp Ther Med 2019;17:3755-67.

23. Hu Q, Luo W, Huang L, et al. Apoptosis-related microRNA changes in the right atrium induced by remote ischemicperconditioning during valve replacement surgery. SCi Rep 2016;6:18959.

24. Wu H, Zhong Q, Wang J, et al. Beneficial effects and toxicity studies of Xian-ling-gu-bao on bone metabolism in ovariectomized rats. Front Pharmacol 2017;8:273.

25. Liu Z, Liu Y, Tu X, et al. High serum levels of malondialdehyde and 8-OHdG are both associated with early cognitive impartment in patients with acute ischaemic stroke. Sci Rep 2017;7:9493.

26. Giasson BI, Ischiropoulos H, Lee VM, et al. The relationship between oxidative/nitrative stress and pathological inclusions in Alzheimer's and Parkinson's diseases. Free Radic Biol Med 2002;32:1264-75.

27. Nunomura A, Perry G, Aliev G, et al. Oxidative damage is the earliest event in Alzheimer disease. J Neuropathol Exp Neurol 2001;60:759-67.

28. Clausen BH, Lundberg L, Yli-Karjanmaa M, et al. Fumarate decreases edema volume and improves functional outcome after experimental stroke. Exp Neurol
2017;295:144-54.

29. Safari A, Fazeli M, Namavar MR, et al. Therapeutic effects of oral dimethyl fumarate on stroke induced by middle cerebral artery occlusion: An animal experimental study. Restor Neurol Neurosci 2017;35:265-74.

30. Kwah LK, Diong J. National Institutes of Health Stroke Scale (NIHSS). J Physiother 2014;60:61.

31. Bertolin M, Van Patten R, Greif T, et al. Predicting cognitive functioning, activities of daily living, and participation 6 months after mild to moderate stroke. Arch Clin Neuropsychol 2018;33:562-76.

32. Mazya MV, Lees KR, Collas D, et al. IV thrombolysis in very severe and severe ischemic stroke: results from the SITS-ISTR registry. Neurology 2015;85:2098-106.

33. Valerio V, Myasoedova VA, Moschetta D, et al. Impact of oxidative stress and protein S-glutathionylation in Aortic value sclerosis patients with overt atherosclerosis. J Clin Med 2019;8:552.

34. Sousa MSB, Alves DVS, Monteiro HMC, et al. Sepsis impairs the propagation of cortical spreading depression in rats and this effect is prevented by antioxidant extract. Nutr Neurosci 2019:1-10. [Epub ahead of print].

35. Guo NF, Cao YJ, Chen X, et al. Lixisenatide protects doxorubicin-induced renal fibrosis by activating $\mathrm{wNF}$ $\kappa \mathrm{B} / \mathrm{TNF}-\alpha$ and TGF- $\beta /$ Smad pathways. Eur Rev Med Pharmacol Sci 2019;23:4017-26.

36. Ye Q, Feng Y, Wang Z, et al. Effects of dietary Gelsemium elegans alkaloids on growth performance, immune responses and disease resistance of Megalobrama amblycephala. Fish Shellfish Immunol 2019;91:29-39.

37. Shichiri $M$. The role of lipid peroxidation in neurological disorders. J Clin Biochem Nutr 2014;54:151-60.

38. Iadecola $\mathrm{C}$. The pathobiology of vascular dementia. Neuron 2013;80:844-66.

39. Niizuma K, Endo H, Chan PH. Oxidative stress and mitochondrial dysfunction as determinants of ischemic neuronal death and survival. J Neurochem 2009;109:133-8.

40. Rasquin SM, Verhey FR, van Oostenbrugge RJ, et al. Demographic and CT scan features related to cognitive impairment in the first year after stroke. J Neurol Neurosurg Psychiatry 2004;75:1562-7.

41. Barba R, Martínez-Espinosa S, Rodríguez-García E, et al. Poststroke dementia: clinical features and risk factors. Stroke 2000;31:1494-501.

42. Shaqiri A, Danckert J, Burnett L, et al. Statistical learning impairments as a consequence of stroke. Front Hum Neurosci 2018;12:339.

43. Deng R, Hua X, Li J, et al. Oxidative stress markers 
induced by hyperosmolarity in primary human corneal epithelial cells. PLos One 2015;10:e0126561.

44. Wakamatsu TH, Dogru M, Matsumoto Y, et al. Evaluation of lipid oxidative stress status in Sjögren syndrome patients. Invest Ophthalmol Vis Sci 2013;54:201-10.

45. Kobayashi M, Yamamoto M. Nrf2-Keap1 regulation of cellular defense mechanisms against electrophiles and reactive oxygen species. Adv Enzyme Regul 2006;46:113-40.

46. Wu X, Yang B, Hu Y, et al. NRF2 Is a Potential Modulator of Hyperresistance to Arsenic Toxicity in Stem-Like Keratinocytes. Oxid Med Cell Longev 2017;2017:7417694.

47. Lastres-Becker I, García-Yagüe AJ, Scannevin RH, et al. Repurposing the NRF2 activator dimethyl fumarate as therapy against synucleinopathy in Parkinson's disease. Antioxid Redox Signal 2016;25:61-77.

48. Wang Q, Chuikov S, Taitano S, et al. Dimethyl fumarate protects neural stem/progenitor cells and neurons from oxidative damage through Nrf2-ERK1/2 MAPK pathway. Int J Mol Sci 2015;16:13885-907.

49. Berkovich R, Weiner LP. Effects of dimethyl fumarate on lymphocyte subsets. Mult Scler Relat Disord 2015;4:339-41.

50. Chaves C, Ganguly R, Ceresia C, et al. Lymphocyte subtypes in relapsing-remittin multiple sclerosis patients

Cite this article as: Hou $\mathrm{X}, \mathrm{Xu} \mathrm{H}$, Chen W, Zhang N, Zhao Z, Fang X, Zhang X, Chen H, Xu Y. Neuroprotective effect of dimethyl fumarate on cognitive impairment induced by ischemic stroke. Ann Transl Med 2020;8(6):375. doi: 10.21037/ atm.2020.02.10 treated with dimethyl fumarate. Mult Scler J Exp Transl Clin 2017;3:2055217317702933.

51. Harmel P, Schlunk F, Harms L. Fulminant rebound of relapsing-remitting multiple sclerosis after discontinuation of dimethyl fumarate: A case report. Mult Scler 2018;24:1131-3.

52. Wallbrecht K, Drick N, Hund AC, et al. Downregulation of endothelial adhesion molecules by dimethylfumarate, but not monomethyl fumarate, and impairment of dynamic lymphocyte-endothelial cell interactions. Exp Dermatol 2011;20:980-5.

53. Gillard GO, Collette B, Anderson J, et al. DMF, but not other fumarates, inhibits $\mathrm{NF}-\kappa \mathrm{B}$ activity in vitro in an Nrf2-independent manner. J Neuroimmunol 2015;283:74-85.

54. Yao Y, Miao W, Liu Z, et al. Dimethyl fumarate and monomethyl fumarate promote post-ischemic recovery in mice. Transl Stroke Res 2016;7:535-47.

55. Sghaier R, Nury T, Leoni V, et al. Dimethyl fumarate and monomethyl fumarate attenuate oxidative stress and mitochondrial alterations leading to oxiapoptophagy in $158 \mathrm{~N}$ murine oligodendrocytes treated with 7 $\beta$-hydroxycholesterol. J Steroid Biochem Mol Biol 2019;194:105432. 